\title{
Medium term treatment of stable stage cystic fibrosis with recombinant human DNase I
}

\author{
Pallav L Shah, Sandra F Scott, Henry J Fuchs, Duncan M Geddes, \\ Margaret E Hodson
}

\begin{abstract}
Background - A phase II multicentre double blind placebo controlled study in 1993 showed that short term treatment (10 days) with recombinant human DNase I (rhDNase) was safe and improved pulmonary function in patients with cystic fibrosis with stable stage lung disease. A six month open label treatment study was conducted in some of the patients who participated in the short term study to assess the medium term effects of rhDNase.

Methods - Patients who completed the phase II study and were stable for 14 days prior to treatment were eligible. They were treated with rhDNase $2.5 \mathrm{mg}$ twice daily for six months and reviewed at regular intervals to assess safety and efficacy.

Results - Fifty nine patients $(31 \mathrm{M}, 28 \mathrm{~F})$ of age range $16-55$ years were recruited. Mean baseline values for forced expiratory volume in one second $\left(F V_{1}\right)$ and forced vital capacity (FVC) were $41.5 \%$ and $72 \cdot 4 \%$ of predicted, respectively. The mean increase in $\mathrm{FEV}_{1}$ over the first month of treatment was $13 \cdot 1 \%$ (range $12-14 \cdot 1 \%$ ) and then stabilised at $6 \cdot 2 \%(4 \cdot 6-7 \cdot 8 \%)$ for the subsequent five months. FVC was similarly improved. Administration of rhDNase improved the severity of dyspnoea, cystic fibrosis related symptoms, and the modified Taussig/NIH score (not statistically significant). Fifty seven of the 59 patients completed the study; two died from progression of their pulmonary disease unrelated to treatment with rhDNase. The adverse events and intercurrent illnesses were no different from those expected in a cystic fibrosis population. Pharyngitis was the only possible drug related adverse event which occurred at least once in $14 \%$ of patients during the six month period.

Conclusions - Administration of rhDNase was safe, well tolerated, and improved pulmonary function in patients with cystic fibrosis. When rhDNase was stopped at day 169 there was a deterioration in pulmonary function and dyspnoea score.

(Thorax 1995;50:333-338)
\end{abstract}

Keywords: cystic fibrosis, recombinant human DNase.
Viscous pulmonary secretions in cystic fibrosis are poorly cleared and this stasis leads to both airways obstruction and increased susceptibility to infection. This creates a self perpetuating cycle of infection and inflammation. The abnormal rheology of the airway secretions is further exaggerated by the inflammatory response. Disintegrating neutrophils release deoxyribonucleic acid (DNA) ${ }^{1}$ which is present in the sputum of patients with cystic fibrosis in concentrations of $3-14 \mathrm{mg} / \mathrm{ml} .^{23}$ The interaction of cystic fibrosis mucoglycoproteins with DNA contributes to the high viscoelasticity of cystic fibrosis secretions. ${ }^{4}$ In addition, neutrophil proteases and oxidants released are responsible for much of the lung damage that occurs. The clinical effects of chronic obstruction of the airways include cough, sputum production, shortness of breath, and a reduction in breathing capacity as measured by pulmonary function tests. Respiratory disease is the major cause of mortality and morbidity in cystic fibrosis. ${ }^{5}$ The median survival in the UK is 24 years $^{6}$ and 27 years in North America. ${ }^{7}$ Reduction of sputum viscoelasticity may have potential benefits in patients with cystic fibrosis, especially by reducing acute and chronic airways obstruction and their subsequent effects.

Human deoxyribonuclease $I$ is an extracellular enzyme produced in the human pancreas and salivary glands. Recombinant human deoxyribonuclease I (rhDNase) was cloned, sequenced, and the expressed product shown to reduce the viscosity of cystic fibrosis sputum in vitro by depolymerising extracellular DNA. ${ }^{8}$ Two phase I studies with rhDNase produced encouraging results ${ }^{910}$ and a phase II multicentre study conducted in the USA showed that rhDNase significantly improved pulmonary function in patients with cystic fibrosis. ${ }^{11} \mathrm{~A}$ parallel phase II double blind placebo controlled short term study with aerosolised rhDNase was conducted at our centre. ${ }^{12}$ Cystic fibrosis patients older than 15 years of age, FVC $>40 \%$ predicted, and oxygen saturations $>90 \%$ were recruited. Seventy one patients with cystic fibrosis were randomised to receive either placebo or rhDNase $2.5 \mathrm{mg}$ twice daily for a period of 10 days and were followed for a further 42 days. The baseline and demographic characteristics of the two groups were similar. 
Treatment with rhDNase significantly improved $\mathrm{FEV}_{1}$ in comparison to placebo $(\mathrm{p}<0.001)$. The magnitude of improvement was $10-15 \%$ (mean change of $13.3 \%$ ) compared with a mean change of $-0.2 \%$ for placebo. The effect on pulmonary function gradually subsided following discontinuation of treatment. FVC was also improved (mean increase $7 \cdot 2 \%$ on rhDNase compared with $2 \cdot 3 \%$ for placebo) but did not reach statistical significance in comparison with placebo. Dyspnoea scores were improved following treatment with rhDNase but these changes were not significant. Overall quality of life scores were not significantly improved. The incidence of adverse events was similar in the two treatment groups. There was no significant change in spirometric values on initial challenge and rechallenge 18 days after the last dose of study drug. No patients developed antibodies to rhDNase.

We now report a six month open label treatment study in these patients in order to evaluate if drug safety and improvements in lung function were maintained over a longer period.

\section{Methods \\ PATIENTS}

Patients who had completed the double blind phase II protocol ${ }^{12}$ were eligible and the minimum time interval between completion of the phase II study and initiation of open label treatment was two months. The patients were required to be outpatients with stable stage pulmonary disease (forced vital capacity (FVC) $>40 \%$ predicted) and to have no qualitative change in their antibiotics, bronchodilators, or steroids within 14 days of enrolment. All women had a negative pregnancy test and were required to take adequate contraceptive precautions throughout the study. The study was approved by the hospital ethical committee and all patients gave informed consent. The double blind code was not disclosed to the patients or investigators until all recruitment was complete. The patients were treated with $2.5 \mathrm{mg}$ rhDNase in $150 \mathrm{mmol} / 1 \mathrm{NaCl}$ plus $1.5 \mathrm{mmol} / 1$ $\mathrm{CaCl}_{2}$ twice daily for six months followed by a two week washout period. All physiotherapy and concomitant medications were maintained and any alterations were recorded.

\section{OUTCOMES}

The determinants of safety and efficacy were identical to those in the double blind protocol. Pulmonary function tests were the primary assessments of efficacy and were performed at each visit according to the American Thoracic Society guidelines. ${ }^{13}$ Intrapatient variability was minimised by reviewing patients at the same time of day throughout the study. Patient questionnaires to assess dyspnoea and quality of $\frac{5}{9}$ life were administered before spirometry at all visits. Patients rated their perception of dyspnoea on a $100 \mathrm{~mm}$ vertical visual analogue $\overrightarrow{\widehat{D}}$ scale $\left(0 \mathrm{~mm}=\right.$ no shortness of breath and $\frac{2}{0}$ $100 \mathrm{~mm}=$ maximum breathing difficulty) ${ }^{14} \mathrm{~A}$ क questionnaire developed during the short term $\overrightarrow{0}$ double blind study was used to evaluate patients' general wellbeing and their cystic fibrosis related symptoms. ${ }^{12}$ General wellbeing was measured according to the response to 0 five questions on feeling, energy level, activity, appetite, and ease of sleeping. Cystic fibrosis related symptoms were scored on ease of sputum expectoration, sense of lung congestion, 우 frequency and severity of cough. Each question $\rightarrow$ was answered on a five point scale $\left(1=\right.$ worst $\frac{7}{O}$ to $5=$ best). A modified NIH score, in which FVC was substituted for slow vital capacity, $\vec{\bullet}$ was also calculated on days 1 and $169 . .^{15}$ of

\section{CONDUCT OF TRIAL (table 1 )}

Initial challenge with $2 \cdot 5 \mathrm{mg}$ rhDNase was conducted under medical supervision and spirometric values were measured both before and $\overrightarrow{\vec{A}}$ 30 minutes after the dose. No patients developed acute sensitivity to rhDNase and therefore all patients were able to continue as outpatients. The drug was delivered using a Pulmo-Aide compressor (DeVilbiss, Somerset, 을 Pennsylvania, USA) and Acorn II jet nebuliser (Marquest, Englewood, Colorado, USA).

At initiation medical history, with details of chest physiotherapy and medications, was recorded. A full physical examination was conducted and routine haematological and biochemical tests were also performed. Serum was obtained for measurement of antibodies to rhDNase (by a radioprecipitation assay ${ }^{16}$ ), and $\widetilde{N}$ women also had a pregnancy test. These tests 0 were repeated on days 29,85 , and 169 . A chest radiograph was taken at initiation and on

\begin{tabular}{|c|c|c|c|c|c|c|c|c|c|c|c|}
\hline \multirow{17}{*}{$\begin{array}{l}\text { Visit number } \\
\text { Study day } \\
\text { Informed consent } \\
\text { Medical history } \\
\text { Physical examination } \\
\text { Vital signs } \\
\text { Spirometry } \\
\text { Modified NIH score } \\
\text { Chest radiograph } \\
\text { Quality of life } \\
\text { Dyspnoea score } \\
\text { Lab safety tests } \\
\text { Serum pregnancy tests } \\
\text { DNase antibodies } \\
\text { Record of adverse event } \\
\text { Review patient diary }\end{array}$} & \multicolumn{11}{|c|}{ Treatment with rhDNase } \\
\hline & 1 & 2 & 3 & 4 & 5 & 6 & 7 & 8 & 9 & 10 & 11 \\
\hline & 1 & 8 & 15 & 29 & 43 & 57 & 85 & 113 & 141 & 169 & 183 \\
\hline & $\mathrm{x}$ & & & & & & & & & & \\
\hline & $\mathbf{X}$ & & & & & & & & & & \\
\hline & $\mathrm{X}$ & & & $\mathrm{X}$ & & & $\mathrm{X}$ & & & $\mathrm{X}$ & \\
\hline & $\mathrm{x}$ & $\mathrm{X}$ & $\mathrm{X}$ & $\mathrm{X}$ & $\mathrm{X}$ & $\mathrm{X}$ & $\mathrm{X}$ & $\mathrm{X}$ & $\mathrm{X}$ & $\mathrm{X}$ & $\mathrm{X}$ \\
\hline & $\mathrm{X}^{\mathrm{a}}$ & $\mathrm{x}$ & $\mathrm{X}$ & $\mathrm{X}$ & $\mathrm{x}$ & $\mathrm{x}$ & $\mathrm{X}$ & $\mathrm{x}$ & $\mathrm{X}$ & $\mathrm{X}$ & $\mathrm{X}$ \\
\hline & $\mathrm{x}$ & & & & & & & & & $\mathrm{X}$ & \\
\hline & $\mathrm{X}$ & & & & & & & & & $\mathrm{X}$ & \\
\hline & $\mathrm{X}$ & $\mathrm{X}$ & $\mathrm{X}$ & $\mathbf{x}$ & $\mathbf{x}$ & $\mathrm{X}$ & $\mathrm{X}$ & $\mathbf{X}$ & $\mathbf{X}$ & $\mathrm{x}$ & $\mathrm{X}$ \\
\hline & $\mathrm{x}$ & $\mathrm{x}$ & $\mathrm{x}$ & $\mathrm{X}$ & $\mathrm{x}$ & $\mathrm{x}$ & $\mathrm{X}$ & $\mathrm{x}$ & $\mathrm{x}$ & $\mathrm{x}$ & $\mathrm{x}$ \\
\hline & $\mathrm{X}$ & & & $\mathrm{X}$ & & & $\mathrm{X}$ & & & $\mathrm{X}$ & \\
\hline & $\mathrm{X}$ & & & $\mathrm{x}$ & & & $\mathrm{X}$ & & & $\mathrm{X}$ & \\
\hline & $\mathrm{X}$ & & & $\mathrm{X}$ & & & $\mathrm{X}$ & & & $\mathrm{X}$ & $\mathrm{X}$ \\
\hline & $x$ & $X$ & $x$ & $\mathrm{X}$ & $\mathrm{X}$ & $\mathbf{x}$ & $\mathrm{X}$ & $\mathrm{X}$ & $\mathrm{X}$ & $\mathrm{X}$ & $\mathrm{X}$ \\
\hline & $\mathrm{x}$ & $\mathrm{x}$ & $\mathrm{x}$ & $\bar{x}$ & $\mathrm{X}$ & $\mathrm{x}$ & $\mathrm{X}$ & $\mathrm{X}$ & $\mathrm{X}$ & $\mathrm{X}$ & $\mathrm{X}$ \\
\hline
\end{tabular}

${ }^{a}$ Spirometric tests repeated 30 minutes after dose. 
Table 2 Mean (SE) and range of demographic and baseline characteristics

\begin{tabular}{ll}
\hline Age (years) & $27 \cdot 5(1 \cdot 1)$ \\
& $16-55$ \\
Sex & $31 \mathrm{M} / 28 \mathrm{~F}$ \\
Height $(\mathrm{cm})$ & $165 \cdot 7(1 \cdot 2)$ \\
& $139-197$ \\
Weight $(\mathrm{kg})$ & $54 \cdot 2(1 \cdot 2)$ \\
FEV $_{1}$ (\% predicted) & $35 \cdot 3-79 \cdot 3$ \\
FVC (\% predicted) & $41 \cdot 5(2 \cdot 4)$ \\
& $17 \cdot 7-99 \cdot 3$ \\
Dyspnoea score (mm) & $72 \cdot 4(3 \cdot 1)$ \\
& $36 \cdot 6-130 \cdot 7$ \\
Wellbeing score & $38 \cdot 4(3 \cdot 2)$ \\
Cystic fibrosis symptom score & $0-92$ \\
NIH score & $3 \cdot 4(0 \cdot 1)$ \\
& $1 \cdot 8-5 \cdot 0$ \\
& $2 \cdot 1(0 \cdot 1)$ \\
\hline
\end{tabular}

$\mathrm{FEV}_{1}=$ forced expiratory volume in one second $\mathrm{FVC}=$ forced vital capacity.

completion of the trial. Patients were asked to keep a diary record of drug administration times and any alteration in medications or chest physiotherapy. Vital signs, lung spirometry, dyspnoea score, general wellbeing score, cystic fibrosis related symptom score, adverse events, and drug accountability were assessed at each visit. Patients were reviewed two weeks after completion of the six month treatment period and pulmonary function, dyspnoea score, quality of life scores, and vital signs were re-evaluated (table 1). Patient diaries were also reviewed and any new adverse events or alterations in treatment recorded. Serum for measurement of rhDNase antibodies was also obtained.
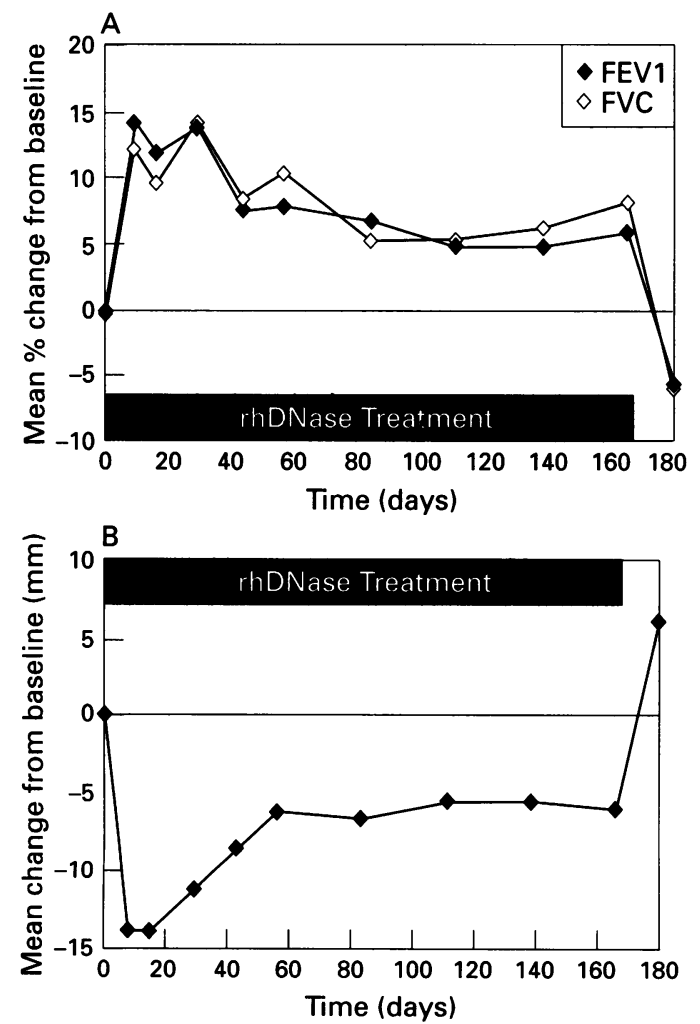

Figure 1 (A) Mean percentage change from baseline in pulmonary function (FEV, and $F V C$ ) and (B) mean change in visual analogue dyspnoea score $(\mathrm{mm})$ following treatment with rhDNase. Treatment was stopped on day 169.
STATISTICAL ANALYSIS

Under the "intention to treat" principle all patients who entered the study were included in the analysis and descriptive statistics used to display the results of open label treatment with rhDNase. Pulmonary function was evaluated by calculating mean percentage changes from baseline which were obtained before initiation of open label treatment. This was defined as:

$$
\frac{\text { Treatment } \mathrm{FEV}_{1}-\text { baseline } \mathrm{FEV}_{1}}{\text { Baseline } \mathrm{FEV}} \times 100
$$

Conversion of pulmonary function variables to mean percentage changes from baseline allowed an assessment of the effect of treatment in a population with heterogeneous values for pulmonary function as suggested by the American Thoracic Society. ${ }^{17}$

The effects of treatment on dyspnoea scores, wellbeing score, and cystic fibrosis related symptom score were also evaluated by examining changes from baseline (pretreatment study day 1 ).

\section{Results}

Fifty nine patients were enrolled from 71 patients who had completed the double blind protocol; 31 had received placebo and 28 rhDNase during the double blind trial. One patient was excluded due to a qualitative change in antibiotic regimen within 14 days before enrolment and the remaining 11 patients

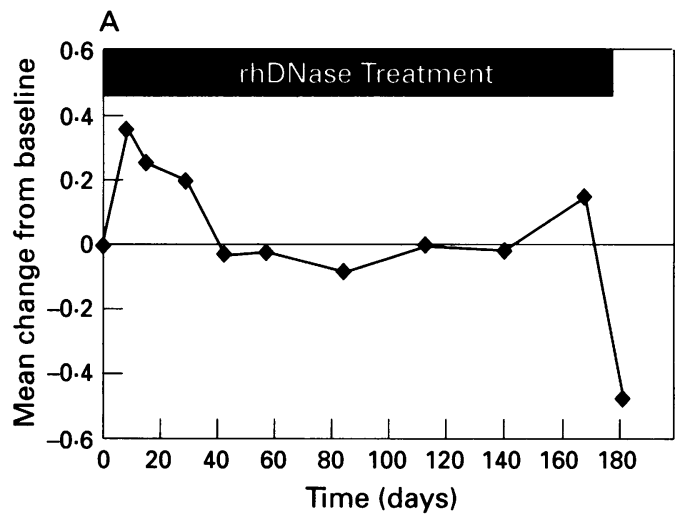

B

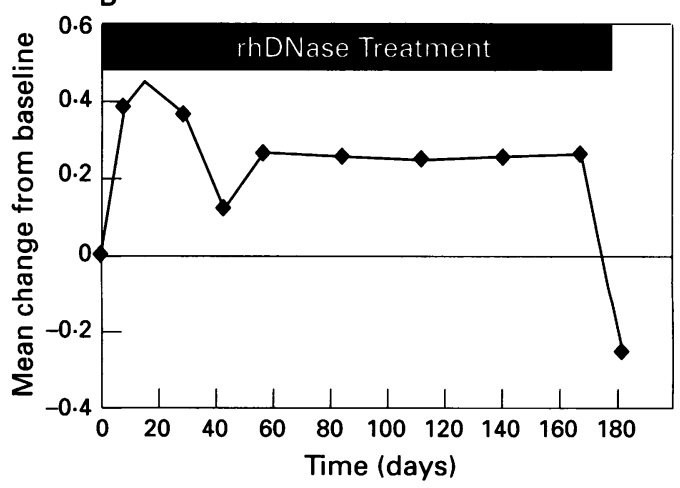

Figure 2 Mean change from baseline in $(A)$ general wellbeing score and (B) cystic fibrosis related symptom score following treatment with rhDNase. Treatment was stopped on day 169. 
Table 3 Summary of serious adverse events

\begin{tabular}{lcc}
\hline Adverse event & No. & $\%$ \\
\hline General & & 2 \\
Chest pain & 1 & 3 \\
Deaths (sepsis, respiratory failure) & 2 & 2 \\
Cardiovascular & 1 & 2 \\
$\quad$ Pericarditis & 1 & 2 \\
Gastrointestinal & 1 & 5 \\
$\quad$ Diarrhoea & 2 & 2 \\
Duodenitis & 3 & 5 \\
Meconium ileus equivalent & 1 & 2 \\
Despiratory & 3 & \\
Haemoptysis & 19 & 1 \\
$\quad$ Pneumothorax & 1 & 2 \\
$\quad$ Exacerbation of respiratory tract infection & & 2 \\
Wheeze &
\end{tabular}

declined to enter the six month study because of the follow up visits required. All patients except two who died completed the six month treatment. The demographic and baseline characteristics are presented in table 2 . All patients were receiving routine chest physiotherapy upon entering into the study. Of the 59 patients $32(54 \%)$ had a baseline FVC value between $40 \%$ and $69 \%$ predicted, $14(24 \%)$ had a baseline FVC between $70 \%$ and $89 \%$, and $13(22 \%)$ had a baseline FVC of $>90 \%$ predicted.

Administration of $2.5 \mathrm{mg}$ rhDNase twice daily improved pulmonary function. The mean increase in $\mathrm{FEV}_{1}$ over the first month of treatment was $13 \cdot 1 \%$ (range $12-14 \cdot 1 \%$ ) which then stabilised at $6 \cdot 2 \%(4 \cdot 6-7 \cdot 8 \%)$ for the subsequent five months (fig 1A). FVC improved by $12 \cdot 0 \%(9 \cdot 6-14 \cdot 3 \%)$ during the first month and then levelled at $7 \cdot 2 \%(5 \cdot 1-10 \cdot 2 \%)$. The effect on pulmonary function subsided with cessation of therapy and two weeks after the last dose of rhDNase the mean FVC was 5.9\% and $\mathrm{FEV}_{1} \quad 5 \cdot 7 \%$ below baseline. Severity of dyspnoea as measured by the visual analogue scale was improved by rhDNase (fig 1B). The greatest improvements were observed over the first four weeks (mean reduction of $9.1 \mathrm{~mm}$ ) followed by a plateau at a lower level (mean reduction of $6.4 \mathrm{~mm}$ ). This appears to correlate with the changes in pulmonary function.

Quality of life questions were grouped into two subscores defined as general wellbeing score and the cystic fibrosis related symptom score. The mean changes from baseline in the wellbeing score at each visit during the six month period are plotted in fig $2 \mathrm{~A}$ where an increase in the score denotes improvement. The greatest improvement in the wellbeing score was observed during the first four weeks of the study and these improvements had returned to baseline by study day 43 (overall change from baseline over six months not statistically significant). There was a trend towards improvement in the cystic fibrosis related symptom score throughout the six month treatment period (fig 2B), the improvements ranging from 0.13 to 0.4 points (overall change not statistically significant). The modified NIH score was improved by 4.9 points by the end of six months and there were no apparent differences in chest radiographs performed on day 1 and day 169 of the study.

No acute adverse events occurred and the initial challenge was well tolerated. Three of the 59 patients (5\%) experienced a $>10 \%$ de- crease in $\mathrm{FEV}_{1}$ (mean decrease of $11.9 \%$ ) and eight of the $59(14 \%)$ experienced a $>10 \%$ decrease in FVC (mean decrease 23.1\%) 30 minutes after the initial dose on study day 1 . The patients were asymptomatic and therefore continued on treatment. Despite this initial transient decrease, lung function was improved compared with pretreatment baseline levels over the remaining months on treatment. The overall mean (SE) percentage change from baseline in $\mathrm{FEV}_{1} 30$ minutes after the initial dose of rhDNase was $-0 \cdot 1(0 \cdot 8) \%$, and mean (SE) change in FVC was $+1 \cdot 4(2 \cdot 6) \%$. No patients experienced a decrease in $\mathrm{FEV}_{1}$ of $>30 \%$ or other reactions suggestive of drug allergy or anaphylaxis over the six month treatment period. Only two patients developed antibodies to rhDNase which were detected at three months but were negative by study days 169 and 183. Neither of these patients developed any allergic reactions over the treatment period, nor was there any correlation between the positive antibody result and the patients' pulmonary function and clinical symptoms. Results of haematological and biochemical tests showed no consistent changes from baseline.

Overall the adverse events and intercurrent illnesses seen were no different from those expected in a cystic fibrosis population. Pharyngitis was the only possible drug related adverse event which occurred at least once in $>10 \%$ of the patients, with a total of 17 episodes in eight (14\%) patients. All but one of these episodes was mild, required no alteration in treatment, and resolved within 21 days of onset. The individual who had a moderately severe episode of pharyngitis responded to a reduction in his rhDNase dose to $2.5 \mathrm{mg}$ once daily for two weeks and was then able to tolerate the twice daily dose without recurrence. The serious respiratory events that occurred over the six month period were mainly patient hospitalisations for exacerbations of respiratory tract infections (table 3). There were three episodes of pneumothoraces, two of which occurred in individuals who had previously discontinued treatment with rhDNase. The incidence of pneumothoraces was similar to that observed in a cystic fibrosis population. One individual developed pericarditis two weeks after the discontinuation of rhDNase (study day 183). The event was suspected to be of viral aetiology and resolved within 10 days of onset.

There were two deaths during the study, both of which were considered to be due to natural progression of their disease. One patient was well until day 51 of the study when he developed features of a respiratory tract infection. He was hospitalised locally and therefore treatment with rhDNase was interrupted. The protocol was not approved at other centres and hospitalisation at any other centre required mandatory discontinuation of treatment with rhDNase. The patient had a poor response to treatment and developed an episode of haemoptysis, intermittent fever, weight loss, increased sputum production, and increased dyspnoea. He was transferred to our centre on day 76 . 
On study day 80 he developed a pneumothorax and continued to deteriorate despite the insertion of an intercostal tube. He died due to respiratory failure on study day 80 . The second patient developed a respiratory tract infection on study day 62 and was hospitalised at an institution abroad. Treatment with rhDNase was discontinued on hospitalisation (study day 73). Her treatment was complicated by the development of a pneumothorax on study day 90 and, following further deterioration, required intubation and ventilation on study day 97. She was transferred to our centre on day 112 where she was diagnosed as having a Pseudomonas septicaemia. Her condition progressed and she died on study day 137.

\section{Discussion}

Medium term treatment with $2.5 \mathrm{mg}$ rhDNase twice daily was associated with an increase in pulmonary function. The initial improvement in $\mathrm{FEV}_{1}$ was $10-15 \%$ which is similar to that observed in the treatment arms of the short term double blind study. ${ }^{12}$ Lung function was subsequently maintained at half the initial rise in pulmonary function - that is, mean percentage change of $6 \%$ above baseline. Due to the open label design the study may be confounded by factors such as frequent follow up, close monitoring, intensive professional input in a possibly highly motivated group of patients. Despite this, the initial observations were similar to those in the double blind studies $^{112}$ and the overall pattern of lung function was similar to the North American phase III study ${ }^{18}$ in which 968 patients with cystic fibrosis were randomised to receive either placebo, rhDNase $2.5 \mathrm{mg}$ once daily, or rhDNase $2.5 \mathrm{mg}$ twice daily for six months. There was a significant increase in $\mathrm{FEV}_{1}$ in the rhDNase treated groups which was maximal in the first month of treatment and then reached a plateau around $6 \%$ for the subsequent five months of the study.

The improvements observed in the dyspnoea score were consistent with changes in pulmonary function. The quality of life scores showed a trend towards improvement in the cystic fibrosis related symptom scores, but the general wellbeing score returned to baseline by day 43 . The questionnaires were identical to those used in the short term protocol, allowing the two studies to be compared directly. Both studies recorded a greater change in the cystic fibrosis related symptom scores than in the general wellbeing scores. The double blind phase III study produced similar results and, because of the larger patient population, these changes were statistically significant. ${ }^{18}$ All the efficacy parameters in this study and the double blind phase III study appear to follow a similar pattern of maximal improvement in the first month and a subsequent plateau at a lower degree of improvement. The cause of this effect is uncertain.

Bovine DNase was associated with severe bronchospasm ${ }^{19}$ and it was therefore important to assess the non-specific sensitivity and immunogenicity of rhDNase. Previous short term double blind trials have shown that rhDNase is safe with no evidence of non-specific sensitivity or development of antibodies to rhDNase. ${ }^{112}$ In this medium term treatment study three patients had a fall in $\mathrm{FEV}_{1}$ of $>10 \%$ (mean $11.9 \%$ ) on initial challenge. These individuals were asymptomatic, tolerated further treatment, and had similar improvements in pulmonary function to the overall population. Two patients temporarily developed antibodies to rhDNase. In the phase III study $4 \%$ of patients treated with rhDNase twice daily developed antibodies to rhDNase. ${ }^{18} \mathrm{~A}$ study conducted with intermittent administration of rhDNase to evaluate maximally its immunogenic and allergic potential treated patients with cystic fibrosis with a cyclical regimen of rhDNase $10 \mathrm{mg}$ twice daily for two weeks followed by a two week washout period..$^{20}$ No patients developed acute adverse reactions, anaphylaxis, or drug allergy during the six month study. Seventeen of the 178 patients who participated in the study developed antibodies to rhDNase. Only six of these remained antibody positive at the end of six months. There was no correlation between clinical symptoms, pulmonary function, and the presence of antibodies to rhDNase. Treatment with rhDNase induced improvements in pulmonary function that were similar in patients with or without an antibody response, indicating that the efficacy of rhDNase was not altered by the immune response. However, patients need to be monitored over longer periods to evaluate whether antibody production becomes more prevalent or clinically significant.

There appeared to be no significant hazards associated with the use of rhDNase over a six month period. Death rates observed during the study were similar to published mortality rates in patients with cystic fibrosis. ${ }^{67}$ The incidence of serious respiratory adverse events is consistent with that observed in a cystic fibrosis population with a similar degree of disease severity. ${ }^{57}$ Patient hospitalisations for respiratory tract infective exacerbations were the predominant adverse event. The phase III study reports a modest reduction in overall infective exacerbations requiring parenteral antibiotics in patients treated with rhDNase (32\% reduction in the group treated with twice daily rhDNase compared with placebo). No changes in the chest radiographs at the end of the study were observed; this is similar to many other treatments known to be effective in cystic fibrosis.

The pulmonary disease in cystic fibrosis is progressive and the major cause of morbidity and mortality. ${ }^{5}$ Medium term treatment with rhDNase demonstrates that treatment is safe and that improvements in lung function are maintained, although at a lower level of around $6 \%$ from baseline.

1 Lethem MI, James SL, Marriott C, Burke JF. The origin of DNA associated with mucus glycoproteins in cystic fibrosis sputum. Eur Respir f 1990;3:19-23.

2 Chernick WS, Barbero GJ. Composition of tracheobronchial secretions in cystic fibrosis of the pancreas and bronchiectasis. Pediatrics 1959;24:739-45.

3 Potter J, Matthews LW, Lemm J, Spector JS. Composition 
of pulmonary secretions from patients with and without cystic fibrosis. Am 7 Dis Child 1960;100:493-5.

4 Lethem MI, James SL, Marriott C. The role of mucous glycoproteins in the rheologic properties of cystic fibrosis sputum. Am Rev Respir Dis 1990;142:1053-8.

5 Penketh ARL, Wise A, Mearns MB, Hodson ME, Batten JC. Cystic fibrosis in adolescents and adults. Thorax 1987; 42:526-32.

6 Dodge JA, Morison S, Lewis PA, Colest EC, Geddes D, Russell G, et al. Cystic fibrosis in the United Kingdom 1968-1988: incidence, population and survival. Pediat Perinatal Epidemiol 1993; 7:157-66.

7 FitzSimmons SC. The changing epidemiology of cystic fibrosis. F Pediatr 1993;122:1-9.

8 Shak S, Capon DJ, Hellmiss R, Marsters SA, Baker CL. Recombinant human DNase I reduces the viscosity of cystic fibrosis sputum. Proc Natl Acad Sci USA 1990;87: 9188-92.

9 Aitken ML, Burke W, McDonald G, Shak S, Montgomery $\mathrm{AB}$, Smith AL. Recombinant human DNase inhalation in normal subjects and patients with cystic fibrosis. A phas I study. $\mathcal{F} A M A$ 1992;267:1947-51.

10 Hubbard RC, McElvaney NG, Birrer P, Shak S, Robinson WW, Jolley C, et al. A preliminary study of aerosolised recombinant human deoxyribonuclease I in the treatment of cystic fibrosis. $N$ Engl f Med 1992;326:812-5.

11 Ramsey BW, Astley SI, Aitken ML, Burke W, Colin AA Dorkin HL, et al. Efficacy and safety of short-term adDorkin HL, et al. Efficacy and safety of short-term ad-
ministration of aerosolised recombinant human deministration of aerosolised recombinant human de-
oxyribonuclease in patients with cystic fibrosis. Am Rev oxyribonuclease in patients
Respir Dis $1993 ; 148: 145-51$.

12 Ranasinha C, Assoufi B, Shak S, Christiansen D, Fuchs
H, Empey D, et al. Efficacy and safety of short-term administration of aerosolised recombinant human DNase I in adults with stable stage cystic fibrosis. Lancet 1993; 342:199-202.

13 American Thoracic Society. Standardisation of spirometry - 1987 update. Am Rev Respir Dis 1987;136:1285-98.

14 Gift AG. Validation of a vertical visual analogue scale as a measure of clinical dyspnoea. Rehab Nurs 1989;14:323-5.

15 Taussig LM, Kattwinkel J, Friedewald WT, di Sant'Agnase PA. A new prognostic score and clinical evaluation system for cystic fibrosis. $\mathcal{F}$ Pediatr 1973;82:380-90.

16 Sinicropi D, Dere R, Shak S. ELISA and radioimmunoprecipitation assays for determination of serum antibody titre to recombinant human DNase. Pediatr Pul- $\overline{\bar{N}}$ monol 1991;Suppl 6:300.

17 American Thoracic Society. Lung function testing: selection of reference values and interpretative strategies. Am Rev Respir Dis 1991;144:1202-18.

18 Fuchs HJ, Borowitz DS, Christiansen DH, Morris EM, Nash ML, Ramsey BW, et al. Effect of aerosolised re- $\mathbb{D}$ combinant human DNase on exacerbations of respiratory symptoms and on pulmonary function in patients with of cystic fibrosis. $N$ Engl f Med 1994;331:637-42.

19 Raskin P. Bronchospasm after inhalation of pancreatic dor- $\vec{\circ}$ nase. Am Rev Respir Dis 1968;98:697-8.

20 Fuchs HJ, Borowitz D, Christiansen D, Morris EM, Nash ML, Ramsey BW, et al. Aerosolised recombinant human DNase reduces pulmonary exacerbations and improves pulmonary function in patients with cystic fibrosis (ab- $\vec{x}$ pulmonary function in patients with cystic fibrosis (abstract). Presented at the 36th Annual Conference on Chest of Disease, Intermountain Thoracic Society, 26 January 1993, Snowbird, Utah, USA. 